Results 110 samples, $\sim 10$ from each CST, were selected. $5.5 \% \quad(\mathrm{n}=6)$ had Amsel-BV, 32.7\% $(\mathrm{n}=36)$ Nugent-BV, and $36.4 \%(\mathrm{n}=40)$ had low-Lactobacillus CSTs (IV, VI, VII); $8.2 \%$ had symptoms. Among Amsel-BV samples, 83.3\% had NugentBV, $16.7 \%$ had intermediate Nugent score and all were CST IV. $86.1 \%$ of women with Nugent-BV and $85 \%$ of women with low-Lactobacillus CSTs did not have Amsel-BV. 22.2\% of those with Nugent-BV did not have low-Lactobacillus CSTs; of these 50\% were CST III (L. iners-dominated). 46.7\% of CST-III had a vaginal $\mathrm{pH} \geq 4.5$, and $13.3 \%$ had a Nugent BV score. 22 samples had a vaginal $\mathrm{pH} \geq 4.5$ and a normal Nugent score: $45.5 \%$ were in CST III, 9.1\% in low-Lactobacillus CSTs. $30.6 \%$ women with Nugent-BV had a vaginal $\mathrm{pH}$ $<4.5$; of these $45.5 \%$ were in CST IV.

Conclusion Nugent score and low-Lactobacillus CST were concordant. L. iners-dominated CSTs often had normal Nugent scores and high $\mathrm{pH}$. Among mostly asymptomatic women, a large proportion with low-Lactobacillus CSTs did not have Amsel-BV. Future studies assessing long term clinical outcomes will be needed to determine whether molecular methods provide added actionable or prognostic information.

Disclosure No significant relationships.

\section{P375 RISK FACTORS FOR INCIDENT BACTERIAL VAGINOSIS AMONG HETEROSEXUAL WOMEN}

${ }^{1}$ Christina Muzny ${ }^{*},{ }^{2}$ Kristal Aaron, ${ }^{1}$ Angela Pontius, ${ }^{1}$ Cheri Aycock, ${ }^{1}$ Jane Schwebke. ${ }^{1}$ University of Alabama at Birmingham, Division of Infectious Diseases, Birmingham, USA; ${ }^{2}$ University of Alabama at Birmingham, Medicine/Infectious Diseases, Birmingham, USA

\subsection{6/sextrans-2019-sti.477}

Background The exact etiology of bacterial vaginosis (BV) is unknown and it is controversial whether BV is a sexually transmitted infection (STI). Gardnerella vaginalis is present in $95-100 \%$ of BV cases. However, it is also found in women with normal vaginal flora and colonization is not sufficient for BV development. We investigated risk factors for incident BV (iBV) in a longitudinal study of heterosexual women.

Methods Women ages 18-45 with no Amsel criteria, Nugent score 0-3, no recent antibiotic use, and no evidence of STI were followed for iBV (Nugent score 7-10 on 3 consecutive days) with daily self-collected vaginal swabs for 8 weeks. Vaginal swabs were also obtained at enrollment for $G$. vaginalisquantitative PCR. Time to iBV was estimated and baseline participant characteristics were compared according to development of iBV or not. All analyses were conducted using SAS v9.4.

Results Between January 2015-May 2018, 164 eligible heterosexual women were enrolled, of which the majority were African American (73\%). Of those enrolled, 29 (17.7\%) developed iBV at a median time of 21 (range 6-50) days. Women with iBV were significantly more likely to be older $(33 \pm 7$ years vs $30 \pm 7$ years; $p=0.02)$, African American $(93 \%$ vs $69 \% ; \mathrm{p}=0.02)$, have a BV history $(72.4 \%$ vs $48.2 \%$; $\mathrm{p}=0.02)$, and have a pregnancy history $(78 \%$ vs $54 \%$; $\mathrm{p}=0.02)$. They were also significantly more likely to have detectable $G$. vaginalis by quantitative PCR at enrollment $(81 \%$ vs $60 \% ; \mathrm{p}=0.04)$ with a higher median number of copies/ $\mu \mathrm{L} \quad(4,850 \quad[1,890-30,100] \quad$ vs $1,340 \quad[217-9,550]$; $\mathrm{p}=0.03)$. No significant difference was found between women with iBV and those without regarding current tobacco use, history of bacterial STI, and lifetime number of sexual partners. Conclusion This study confirms previous cross-sectional findings of risk factors for $\mathrm{iBV}$ among heterosexual women and examines the potential role of $G$. vaginalis in $\mathrm{iBV}$ pathogenesis.

Disclosure No significant relationships.

\section{P380 THE ASSOCIATION BETWEEN SOCIAL SUPPORT, VIOLENCE EXPERIENCE, AND SOCIAL SERVICE NEEDS AMONG A SELECT SAMPLE OF URBAN ADULTS}

${ }^{1}$ Aruna Chandran*, ${ }^{2}$ Amanda Long, ${ }^{3}$ Yvonne Kingon, ${ }^{2}$ Jeannie Murray, ${ }^{4}$ Errol Fields, ${ }^{4}$ Christina Schumacher, ${ }^{3}$ Adena Greenbaum, ${ }^{2}$ Jacky Jennings. ${ }^{1} J o h n s$ Hopkins Bloomberg School of Public Health, Epidemiology, Baltimore, USA; ${ }^{2} J o h n s$ Hopkins University School of Medicine, Center for Child and Community Health Research (CCHR), Baltimore, USA; ${ }^{3}$ Baltimore City Health Department, Baltimore, USA; ${ }^{4}$ Johns Hopkins School of Medicine, Baltimore, USA

\subsection{6/sextrans-2019-sti.478}

Background Experiencing violence is associated with negative health outcomes including increased substance use, mental health problems, and sexual risk behaviors. Studies show associations between strong perceived social support and decreased negative health outcomes. The objectives of this study were: (1) determine the impact of violence (exposure and victimization) on social service needs and perceived health status, and (2) assess whether social support is associated with decreased violence experience among a select sample of urban adults.

Methods A cross-sectional survey was conducted among 187 adults seen in one of 7 urban clinics as part of a larger project between September 2015 and March 2018. A multivariable logistic regression model was used, adjusting for the clinic site as well as age and gender identity.

Results 131 (72\%) individuals had ever witnessed and 89 (49\%) had ever experienced physical assault. Violence victimization was associated with an 8.6-fold odds (95\% CI: 2.4, 31.4) of needing housing assistance and 3.4-fold increased odds (95\% CI: $1.4,8.5$ ) of needing mental health/substance use support services. Exposure to violence was associated with a 5.2-fold increased odds (95\% CI: 2.0, 13.4) of needing housing assistance, a 12.4-fold increased odds (95\% CI: 2.1, 74.0) of needing transportation support, and a 0.5 decreased odds (95\% CI: $0.3,0.8$ ) of reporting optimal health status. Increased perceived social support was associated with 0.54 odds (95\% CI: $0.4,0.8$ ) and 0.62 odds (95\%CI: $0.4,0.9)$ of violence exposure and victimization, respectively.

Conclusion Violence experiences were common in this sample of urban adults, and were associated with increased social service needs and suboptimal perceived health status. Augmenting sources of social support is one possible mechanism for addressing these issues. Including care for the effects of experiencing violence as well as social service needs are important in optimizing the health of key populations.

Disclosure No significant relationships. 